\title{
Structural insight on the mechanism of an electron-bifurcating [FeFe] hydrogenase
}

\author{
Chris Furlan ${ }^{1 \dagger}$, Nipa Chongdar ${ }^{2 \dagger}$, Pooja Gupta ${ }^{1}$, Wolfgang Lubitz ${ }^{2}$, Hideaki Ogata ${ }^{3}$, James N. \\ Blaza $^{1 *}$, James A. Birrell ${ }^{2 *}$
}

\begin{abstract}
${ }^{1}$ Structural Biology Laboratory and York Biomedical Research Institute, Department of Chemistry, The University of York; Heslington, York, YO10 5DD, UK.

${ }^{2}$ Max Planck Institute for Chemical Energy Conversion; Stiftstrasse 34-36, 45470 Mülheim an der Ruhr, Germany. ${ }^{3}$ Institute of Low Temperature Science, Hokkaido University, Kita19 Nishi8, Kita-ku, 060-0819 Sapporo, Japan. $\S$ Present address: CSIR-National Institute of Oceanography, Dona Paula - 403004, Goa, India

*Corresponding authors. Emails: jamie.blaza@york.ac.uk, james.birrell@cec.mpg.de

$\dagger$ These authors contributed equally.
\end{abstract}

\begin{abstract}
$\underline{\text { Abstract }}$
Electron-bifurcation is a fundamental energy conservation mechanism in nature. The electronbifurcating $[\mathrm{FeFe}]$ hydrogenase from Thermotoga maritima $(\mathrm{Hyd} \mathrm{ABC})$ requires both NADH and ferredoxin to reduce protons generating hydrogen. The mechanism of electron-bifurcation in HydABC remains enigmatic primarily due to the lack of structural information. Here, we present a $2.3 \AA$ electron cryo-microscopy structure of HydABC. The structure is a heterododecamer composed of two independent 'halves' each made of two strongly interacting HydABC trimers electrically connected via a [4Fe-4S] cluster, forming a bus-bar system. Symmetry expansion identified two conformations: a "closed bridge" and an "open bridge" conformation, where a $\mathrm{Zn}^{2+}$ site may act as a "hinge" allowing domain movement. Based on these structural revelations, we propose two new mechanisms of electron-bifurcation in HydABC.
\end{abstract}

\section{Introduction}

Electron bifurcation channels a pair of electrons from a single two-electron donor to two different spatially separated electron acceptors with one being at a lower redox potential than the donor (1). This process drives thermodynamically unfavorable (endergonic) redox reactions by directly coupling them to energetically favorable (exergonic) redox reactions and, hence, it represents an alternative energy coupling mechanism to the well-known chemiosmotic coupling principle (2). Electron bifurcation was first described in the Q-cycle of the respiratory complex III where the two electrons originating from the oxidation of ubiquinol are bifurcated via a high potential pathway to cytochrome c, and via a low potential pathway to reduce ubiquinone to ubiquinol (3, 4). Electron bifurcation has recently been discovered in a number of other enzymes where an exergonic electron transfer process is used to drive an endergonic one (5-7). Many of these enzymes have been proposed to utilize flavin-based electron bifurcation (FBEB), in which a flavin mononucleotide (FMN) or flavin adenine dinucleotide (FAD) cofactor serves as the branch point for electrons. It first accepts a hydride from an intermediate potential redox couple (typically $\mathrm{NAD}(\mathrm{P}) \mathrm{H})$ and then sends one electron down a high potential pathway, generating an unstable, low potential semi-reduced FMN, with enough reducing power to send the second electron down a low potential pathway. However, the detailed mechanisms by which coupling is achieved are in many cases unknown. The trimeric [FeFe] hydrogenase, HydABC, from Thermotoga maritima accepts electrons from the one-electron carrier ferredoxin $\left(E_{\mathrm{m}}=-453 \mathrm{mV}\right.$ at $\left.80{ }^{\circ} \mathrm{C},(8)\right)$, which is reduced during pyruvate metabolism, and the two-electron carrier $\mathrm{NADH}\left(E_{\mathrm{m}}=-320 \mathrm{mV}\right.$ at $\mathrm{pH} 7$, $(8)$ ), produced during glucose metabolism, to reduce protons to hydrogen $\left(E^{\mathrm{o}}=-420 \mathrm{mV}\right.$ at $\mathrm{pH} 7$, (8)). The mechanism by which this enzyme functions is debated, however, the predominant view 
is that an FBEB mechanism is operative (9). The biochemically characterized FMN center, present at the NADH binding site is unlikely to act as a bifurcating center because it accepts two electrons from the most oxidizing redox couple $\left(\mathrm{NADH} / \mathrm{NAD}^{+}\right.$) and would then need to bifurcate to two lower potential redox couples. Therefore, the site of bifurcation has been speculated to be a second flavin cofactor (10). However, biochemical studies do not corroborate the presence of the second flavin (11). In another hypothesis, the hydrogen conversion center, the so-called H-cluster, which also undergoes two-electron redox chemistry, is speculated to be the electron-bifurcation center (4). However, spectroscopic studies suggest that the H-cluster of HydABC has similar redox properties to the non-bifurcating $[\mathrm{FeFe}]$ hydrogenases, having a stable one-electron reduced state, and is, therefore, also unlikely to be the site of bifurcation (11). As structural data would reveal the complex arrangement of redox cofactors in this enzyme and provide a stronger basis for understanding the mechanism of electron bifurcation, here we report a $2.3 \AA$ resolution structure of HydABC based on electron cryo-microscopy (cryo-EM) of single particles.

The cryo-EM structure suggests a synergic coupling between two HydABC trimers connected through the His-ligated [4Fe-4S] cluster in the HydA subunit, acting as an electronic bus-bar (12). The structure also reveals flexible C-terminal (CT) domains in HydA and HydB (here named "bridge" domains), which contain additional iron-sulfur clusters that interact through non-covalent interactions and may provide a second electron transfer pathway. Thus, this structure provides details of the arrangement of the redox clusters in HydABC, based on which a novel mechanism of electron-bifurcation is proposed.

\section{$\underline{\text { Results }}$}

\section{The structure of HydABC}

The heterologous production of apo-HydABC in Escherichia coli was described recently (11). Here, we have used this heterologously expressed HydABC to prepare the cryo-EM grids. Following the grid imaging, data collection, and processing, we obtained a $2.3 \AA$ resolution map when a D2 symmetry was enforced (fig. S1, fig. S2, A). Into this, an atomic model of HydABC was constructed, starting with a homology model based on bacterial complex I $(11,13)$, together with $a b$ initio model building in regions of the highest resolution. Initially, the last 91 and $61 \mathrm{C}$ terminal (CT) residues of HydA and $\mathrm{HydB}$, respectively, could not be built as they were not present in the homology model and had a low resolution in the map, indicating regions of high heterogeneity (explored later).

The processed cryo-EM map shows that the heterotrimeric HydABC forms a tetrameric complex, $\mathrm{Hyd}(\mathrm{ABC})_{4}$ of HydABC trimer units (protomers). Oligomerization of HydABC occurs through interactions between four HydA subunits in the core of the complex (Fig. 1, A). HydB and HydC extend outward from the core and form the four lobes clearly visible in the 2D class averages (fig. S3). While HydB is most tightly bound to a single HydA (buried surface area of 1,232 $\AA^{2}$, table $\mathrm{S} 1$ ), there are also two salt bridge interactions between HydB-H516 and HydB-K523 to E212 and E215 of a noncognate HydA. Each HydA has extensive, and presumably tight, interaction with one adjacent HydA chain $\left(2,280 \AA^{2}\right)$, and minor, presumably loose interaction with another HydA chain $\left(780 \AA^{2}\right.$ ) (Fig. 1, A). This observation implies that the core of $\mathrm{Hyd}(\mathrm{ABC})_{4}$ is formed by a pair of tightly bound HydA dimers, which have weak interactions with one another. The HydA core is the best resolved part of the map, consistent with the idea of the core being rigid and homogenous (fig. S4). A large cross-shaped solvent channel runs through the center of the $\mathrm{HydA}_{4}$ core, providing access for $\mathrm{H}_{2}$ and $\mathrm{H}^{+}$to the active site for $2 \mathrm{H}^{+} / \mathrm{H}_{2}$ interconversion (the H-cluster) (Fig. 1, A). 

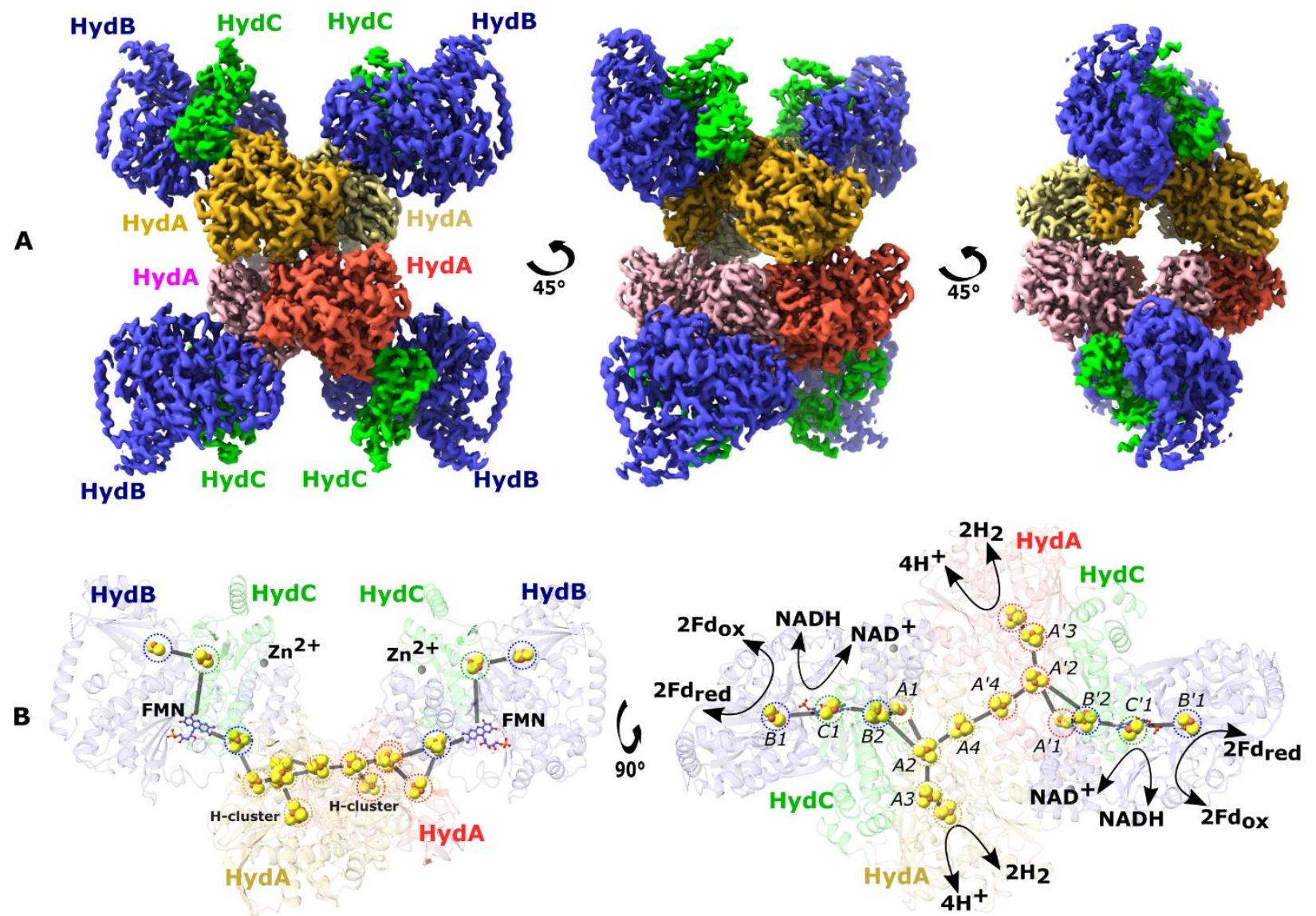

C
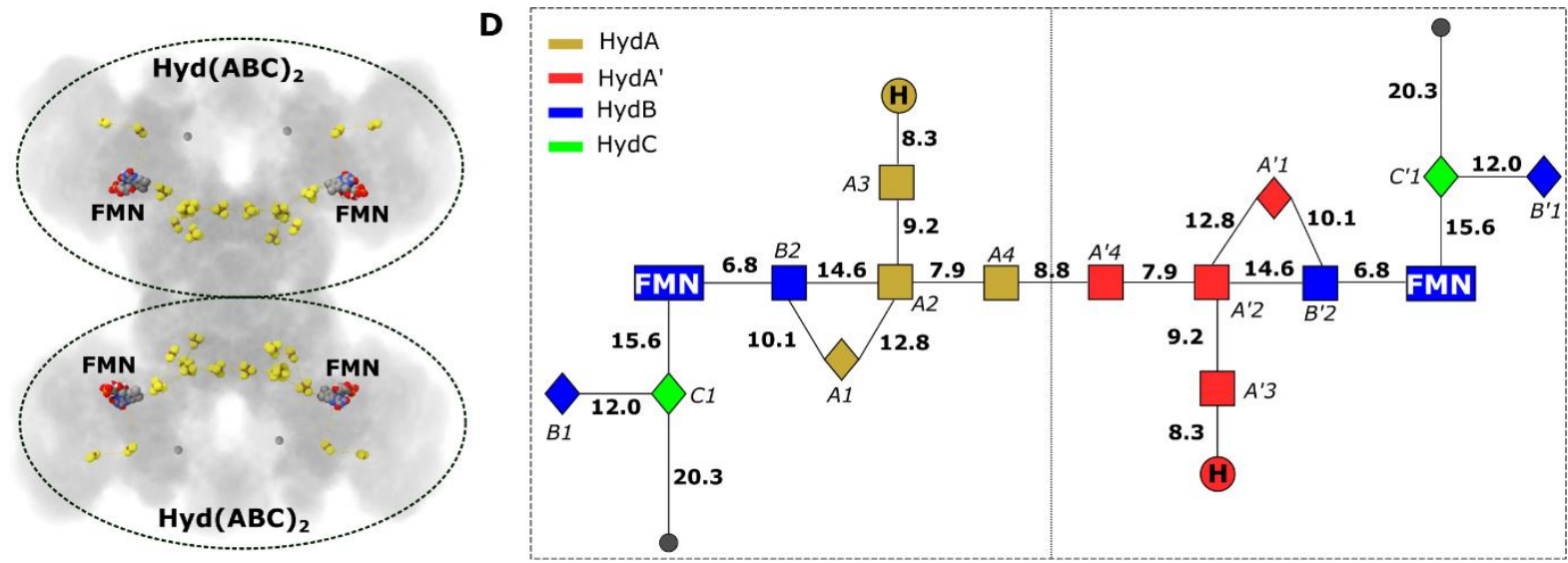

(H) H-cluster $\diamond[2 \mathrm{Fe}-2 \mathrm{~S}] \square\left[\right.$ [4Fe-4S] $\bullet \mathrm{Zn}^{2+}$

Fig. 1. (A) The unsharpened $2.3 \AA$ map of $\mathrm{Hyd}(\mathrm{ABC})_{4}$ with $\mathrm{D} 2$ symmetry enforced showing a tetramer of HydABC trimers. All four copies of HydB and C are colored blue and green, respectively. The four HydA copies that make up the core of the complex are in orange, yellow, pink, and red. The top and bottom halves of the complex are constituted by dimers of $\mathrm{Hyd} \mathrm{ABC}$ protomers (each $\mathrm{HydABC}$ unit is a protomer); the two protomers within the same dimer are strongly interacting, while a weaker interaction is present between the top and bottom dimers. (B) HydABC dimer highlighting the iron-sulfur clusters and FMN constituting the electron transport network. (C) The arrangement of redox cofactors within the protein complex, showing two independent identical redox networks (dashed circles); each redox network is composed of iron-sulfur clusters belonging to a $\mathrm{Hyd}(\mathrm{ABC})_{2}$ unit composed of two strongly interacting HydABC protomers. (D) Schematic of the electron transfer network of one of the two identical Hyd(ABC) 2 units showing edge-to-edge distances (in $\AA$ ) between the various cofactors. 
Each HydABC protomer appears to contain nine redox cofactors including five [4Fe-4S] clusters (one of which forms the [4Fe-4S] subcluster of the H-cluster), three [2Fe-2S] clusters, and one FMN. However, based on Fe quantitation as well as prediction from sequence analysis we expect a total of seven [4Fe-4S] clusters (including the subcluster of the $\mathrm{H}$-cluster) and four [2Fe-2S] clusters in each HydABC protomer $(14,15)$. As per sequence predictions, these missing clusters should be located in the less well resolved CT regions of the HydA and HydB subunits (discussed below) (14). Interestingly, a high-density site, likely a monometallic center, is found in the resolvable part of the HydB-CT domain. Inductively-coupled plasma mass spectrometry on the $\mathrm{HydB}$ subunit identified $\approx 1.5 \mathrm{Zn} /$ protein and $\approx 15 \mathrm{Fe} /$ protein, with $<0.1 \mathrm{Co}, \mathrm{Ni}$ or $\mathrm{Cu}$. As the observed Fe-content matches with the estimated Fe-content of $\mathrm{HydB}$, which is expected to contain three $[4 \mathrm{Fe}-4 \mathrm{~S}]$ clusters and one $[2 \mathrm{Fe}-2 \mathrm{~S}]$ cluster (14 Fe/protein), these results allow us to assign the metal center as zinc $\left(\mathrm{Zn}^{2+}\right)$. This is further supported by the identities of the ligating residues: three cysteines and one histidine in a tetrahedral coordination geometry (fig. S5, C) (16).

\section{Cofactor arrangement in $\mathrm{HydABC}$}

Electron transfer chains, often connecting distant active sites, are composed of redox-active cofactors usually less than $14 \AA$ apart to allow sufficiently fast electron tunnelling through the protein dielectric to sustain catalysis (17). In each HydABC heterotrimer, the spatially distant $\mathrm{H}-$ clusters and FMN centers are electrically connected via a chain of four FeS clusters (A1, A2, A3, and B2, see Fig. 1, D for cluster nomenclature). The edge-to-edge distances between all these clusters are $<15 \AA$ and within a distance for electron transfer at physiologically relevant rates (Fig. 1, D). Among the three remaining FeS clusters, the [4Fe-4S] cluster from HydA (A4) lies at the interface of the two tightly interacting HydA chains, and the two [2Fe-2S] clusters from HydC (C1) and HydB (B1) subunits lie in the vicinity, but on the opposite side, of the FMN. We speculate that this pathway may be connected to the ferredoxin binding site, which would then be located in $\mathrm{HydB}$. Inspection of the global structure of HydB shows a dangling helix at the N-terminus (fig. S6). We speculate that this helix may act as a "fishing rod" for catching ferredoxin similar to a previous "fly casting" mechanism suggested for cyanobacterial complex I (18) and ferredoxin $\mathrm{NADP}^{+}$reductase $(19,20)$. Consistent with this proposal, the closely related formate dehydrogenase, which does not bind ferredoxin, is specifically missing this helix (21). Electrons from ferredoxin would then be transferred to $\mathrm{C} 1$, and possibly to the FMN. However, the distance between $\mathrm{C} 1$ and FMN is $\sim 16 \AA$, which is at the theoretical limit for fast electron transfer (17).

Within the Hyd(ABC) 4 complex, there appear to be two redox networks, each composed of two electrically connected HydABC protomers, separated by at least $50 \AA$ and held together by the strong HydA-HydA interactions (Fig. 1, B and C). The large distance between each network indicates there is no possibility for electrons to be exchanged and that they probably function independently (Fig. 1, C). The two tightly interacting HydABC protomers within the Hyd(ABC) 2 unit are electrically connected through the His-ligated [4Fe-4S] cluster (A4) in HydA (Fig. 1, B), part of the so-called Y-junction (22). These two clusters are separated by $9.0 \AA$ and have the possibility to act as an electronic bus-bar: allowing overflow of electrons from one protomer to the other. An electrical connection between two identical protomers has already been observed in cytochrome $b c_{1}$ (12) for which the bus-bar is speculated to have a number of possible roles such as allowing the physiological function of the protein even after operational damage of one of the two protomers.

The spatial arrangement of subunits HydA, B and C in the HydABC protomer is similar to that of subunits Nqo3, Nqo1, and Nqo2, respectively, in Thermus thermophilus (Tt) respiratory complex I (fig. S7). Furthermore, the individual subunits show a high degree of structural homology, with 
the highest similarity between HydB and Nqo1 (rmsd $1.040 \AA$ ) (23), followed by HydC and Nqo2 (rmsd $1.152 \AA$ ) and the lowest similarity between HydA and Nqo3 (rmsd 1.294 $\AA$ ) (Fig. 2, A). The remarkable structural similarities between $\mathrm{HydB}$ and Nqo1 subunits agree with their common evolutionary origins (24), and suggest that NADH oxidation follows a similar mechanism in both enzymes (Fig. 2, B). The structural differences between Nqo3 and HydA likely reflect the fact that the latter accommodates the hydrogenase $\mathrm{H}$-cluster and facilitates oligomerization of the Hyd $(\mathrm{ABC})_{4}$ complex.

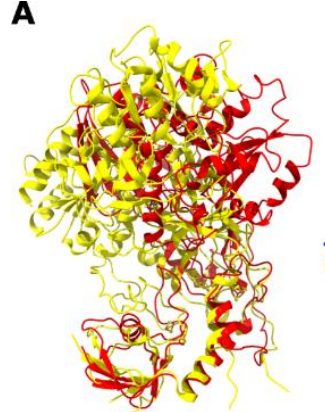

HydA, Nqo3

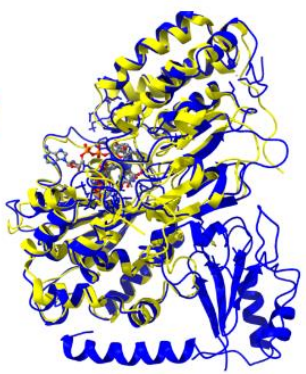

HydB, Nqo1

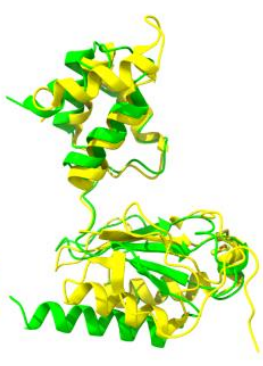

HydC, NqO2
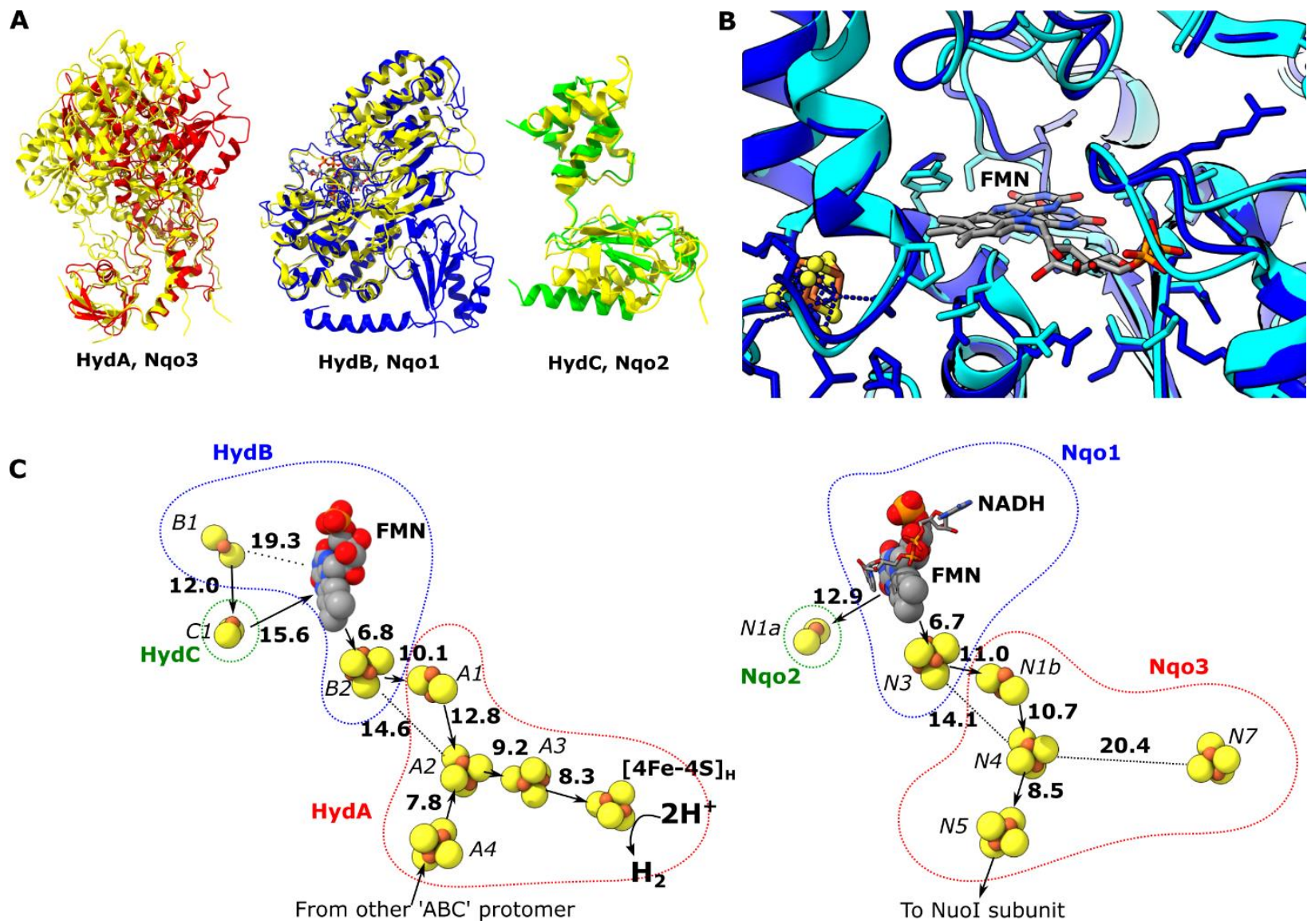

Fig. 2. (A) Subunits HydA (red), HydB (blues) and HydC (green) overlaid with, respectively, Nqo3, Nqo1, Nqo2 (all yellow) of complex I from T. thermophilus (ref.pdb 6ZIY). (B) Comparison of the NADH binding site of the Nqo1 subunit of complex I from Thermus Thermophilus (light blue) with the FMN site in HydB; the high similarity suggests $\mathrm{NADH}$ binds in the proximity of FMN in HydABC similarly to complex I. (C) Electron transfer network in HydABC compared to complex I from $T$. thermophilus.

The structural similarities between $\mathrm{HydABC}$ and $T t$ respiratory complex I is also reflected by the FeS clusters positioning that is in excellent agreement in these two proteins (Fig. 2, C). However, in contrast to the $T t$ complex I, the HydABC protomers contain five additional FeS clusters. One of these additional clusters is a [4Fe-4S] cluster (A3) that electrically connects the [4Fe-4S] subcluster of the H-cluster (analogous to the cluster N7 in Tt complex I) with the rest of the electron transfer network. Another additional cluster is a [2Fe-2S] cofactor in $\mathrm{HydB}(\mathrm{B} 1)$ that is electrically connected to the [2Fe-2S] cluster in $\mathrm{HydC}(\mathrm{C} 1$, analogous to $\mathrm{N} 1 \mathrm{a}$ in $T t$ complex $\mathrm{I})$; due to this connection and the proximity of HydC to the "bridge" domains (discussed later) it is likely that the $[2 \mathrm{Fe}-2 \mathrm{~S}]$ cluster in HydC has an important role in the mechanism, this is in contrast to its 
analogous N1a cluster in complex I, the role of which is unclear but is certainly not part of the main catalytic electron transport pathway from FMN to the quinone binding site $(25,26)$.

HydA has $35 \%$ sequence identity to the monomeric non-bifurcating [FeFe] hydrogenase from Clostridium pasteurianum, CpI. Aligning the two enzymes shows high similarity (rmsd $1.119 \AA$ ) and excellent conservation of the FeS clusters, including the cluster comprising the bus-bar in HydA (Fig. 3). However, in $C p$ I the cluster homologous to A4 is thought to lead to the ferredoxin binding site (27). The multimerization of HydA blocks this site, so the two enzymes must have different ferredoxin binding sites. This re-arrangement shows how closely related systems may have different electron-transfer pathways formed by different multimerization of their subunits.

A

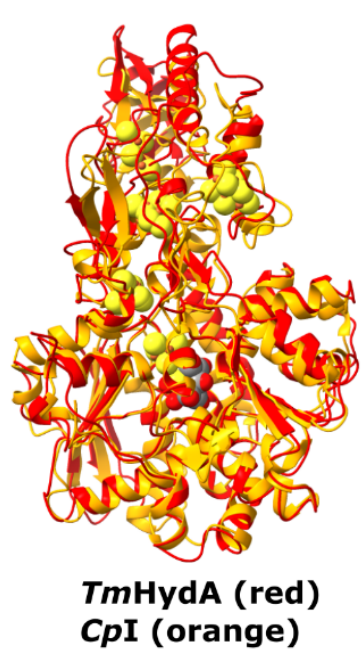

B

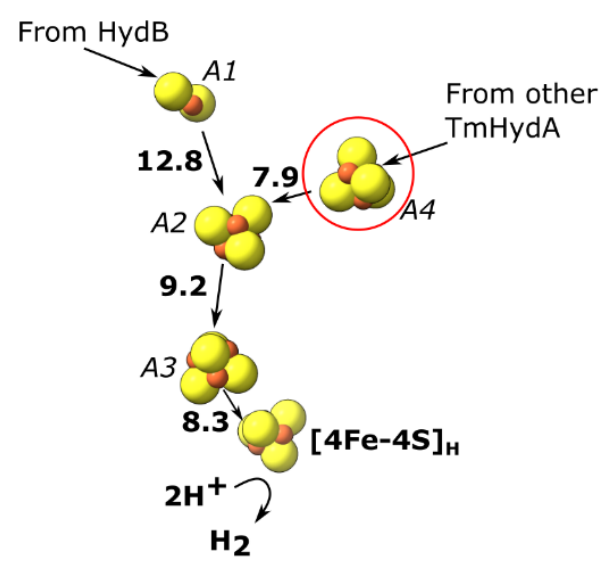

TmHydA
C

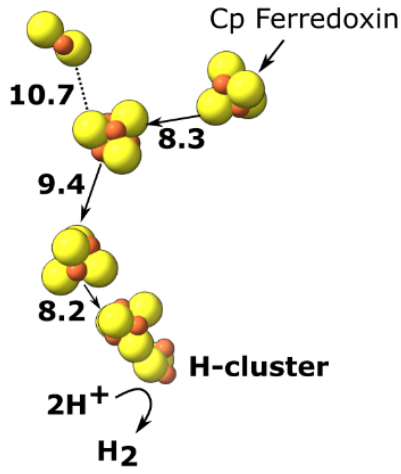

CpI

Fig. 3 (A) HydA from Thermotoga maritima (red) compared with $C p$ I hydrogenase from Clostridium pasteurianum (orange). (B) Electron transfer network in HydA showing the iron-sulfur cluster involved in the bus-bar (red circle). (C) Electron transfer network in $C p \mathrm{I}$, with $C p$ ferredoxin, predicted to bind closely to the iron-sulfur cluster on the right (27). Note that only the $[4 \mathrm{Fe}-4 \mathrm{~S}]_{\mathrm{H}}$ subcluster of the $\mathrm{H}$-cluster is present in our $\mathrm{TmHydA}$ structure, whereas the complete $\mathrm{H}$-cluster including the $[2 \mathrm{Fe}]_{\mathrm{H}}$ subcluster is present in the $C p \mathrm{I}$ structure.

\section{A bridging domain formed by the flexible C-termini of the $\mathrm{HydA}$ and $\mathrm{HydB}$ subunits}

The core of the tetrameric HydABC complex is very well resolved, reaching a local resolution of $2.2 \AA$. However, the lobes formed from HydA and HydB subunits have substantially lower local resolution $(\sim 3 \AA)$, due to increased heterogeneity (fig. S4) and low intensity, blurred map density was observed between the lobes of electrically connected HydABC protomers (fig. S8, A). To investigate the blurred regions, symmetry expansion followed by classification was explored to separate the different conformations into classes. Initial attempts to use D2 symmetry, to match the core, resulted in maps no better than before, however, using $\mathrm{C} 2$ symmetry revealed two classes with bridging density between the HydB lobes (Fig. 4, A) with local resolution similar to the lobes formed from $\mathrm{HydA}$ and $\mathrm{HydB}$ (Fig. 4, B). This bridging density breaks the rotational symmetry between the protomers in the $\mathrm{HydABC} 2$ dimer, explaining why D2 symmetry expansion was ineffective. The two classes correspond to the bridge domain being formed between different HydB lobes: when rotated by $180^{\circ}$, the bridges are identical (Fig. 4, A and C). Despite extensive attempts, we were unable to find a class with both bridges in the closed conformation. The observation that both bridges cannot close simultaneously suggests that these behave as reciprocating elements similarly to the Rieske domain in the bifurcating $b c_{1}$ complex (28). 

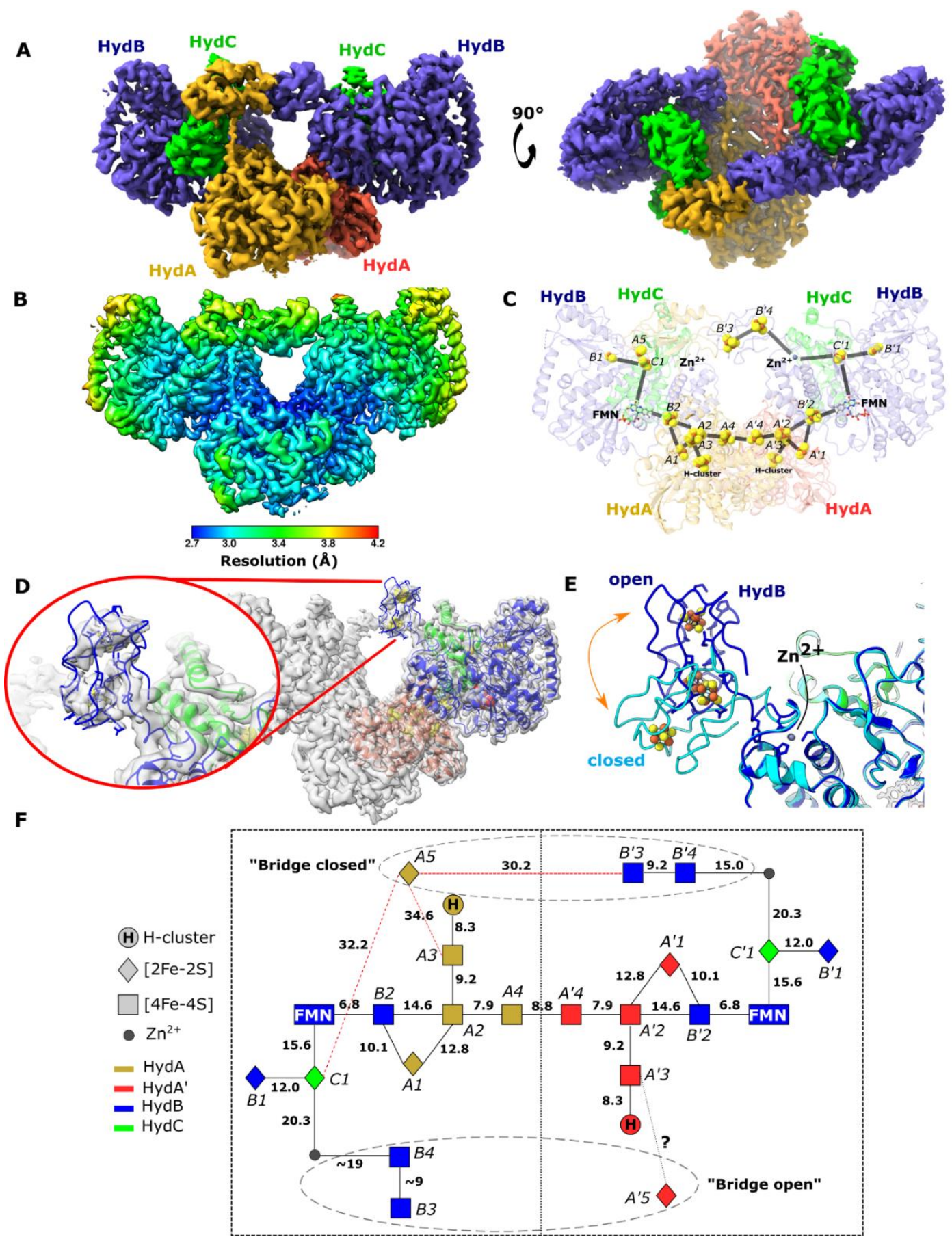

Fig. 4 (A) The unsharpened $2.8 \AA$ map of the bridge forward class sub-particle, identical to the bridge backward class if a $\mathrm{C} 2$ rotation is applied. The map shows only the $\operatorname{Hyd}(\mathrm{ABC})_{2}$ unit as the two $\mathrm{Hyd}(\mathrm{ABC})_{2}$ units constituting the $\mathrm{Hyd}(\mathrm{ABC})_{4}$ complex were found to be independent after 3D classification. All four copies of HydB and C are colored blue and green, respectively. The two HydA copies are in light brown and light red. (B) Local resolutions were estimated using the local resolution function in RELION with default parameters. (C) The atomic model that was built into the map density with the iron-sulfur electron transfer chain. (D) Map showing the HydB bridge domain in the open position and its fitted model. (E) $\mathrm{Zn}^{2+}$ hinge region, showing the two possible conformations of the HydB bridge domain, open (blue) and close (light blue). (F) Schematic of the electron transfer network of one of the two identical $\operatorname{Hyd}(\mathrm{ABC})_{2}$ units showing edge-to-edge distances $(\AA)$ between the components. Represented are the iron-sulfur clusters, H-cluster, FMN and $\mathrm{Zn}^{2+}$ site; the bridge components and $\mathrm{Zn}$-site are enclosed in a dashed ellipse. Each of the two HydABC protomers constituting the $\mathrm{Hyd}(\mathrm{ABC})_{2}$ unit is included within a dashed rectangle. Here the top bridge is represented in its closed conformation, while the bottom one is in its open conformation. 
To further explore the particles without a bridge a further classification was used (Fig. S8, B). It was possible to obtain a low-resolution map of a class where the HydB CT domain was found in an 'open' conformation (Fig. 4, D). Most likely this domain is highly flexible and this conformation is one of many. The movement of the HydB C-terminal domain between the bridgeopen and bridge-closed classes is shown in Fig. 4, E.

In the bridge-containing structure, the two C-terminal [4Fe-4S] clusters (named B3 and B4, Fig. $4, \mathrm{~F})$ of $\mathrm{HydB}$ are close enough to exchange electrons with each other but are too far from the next nearest FeS clusters, such as cluster $\mathrm{C} 1(\approx 35 \AA$ away) or cluster $\mathrm{A} 5(\approx 32 \AA$ away). Furthermore, cluster A5 is completely electrically isolated with all the nearest clusters being $>30 \AA$ away. Thus, unless the HydA and HydB bridge domains undergo substantial conformational changes, the FeS clusters A5, B3 and B4 cannot participate in electronic exchange with the rest of the enzyme.

The bridge structure is particularly interesting as it appears that the $\mathrm{C}$-terminal cysteine residues of $\mathrm{HydB}$ responsible for coordinating [4Fe-4S] clusters in the bridge are conserved in all biochemically characterized electron-bifurcating [FeFe] hydrogenases $(29,30)$. However, they all lack the analogous part of the bridge domain in $\mathrm{HydA}$, which contains the A5 cluster.

\section{$\underline{\text { Discussion }}$}

The importance of flavin based electron bifurcation (FBEB) in microbial metabolism and energy conservation is well acknowledged, but its mechanism is still poorly understood, with only a few examples so far being studied in detail, such as butyryl-CoA dehydrogenase-electron-transferring flavoprotein complex (Bcd-EtfAB) and Fd-dependent transhydrogenase (NfnI) (31). In these enzymes, a specialized flavin center exhibiting 'crossed-over' potentials (i.e., it forms a highenergy one-electron reduced state that can reduce a low potential electron acceptor) functions as the electron bifurcation site. FBEB was also suggested to be operative in electron bifurcating $[\mathrm{FeFe}]$ hydrogenases, which were hypothesized to contain two flavin centers, one functioning as the electron bifurcation site (10). Our cryo-EM structure of HydABC reveals for certain that only a single flavin (the FMN in HydB) that accepts a hydride from NADH, exists in this enzyme.

The H-cluster was also speculated to be an electron-bifurcation center (4). We previously suggested, based on spectroscopic data and redox titrations, that the H-cluster in HydABC behaves similarly to the H-cluster from non-bifurcating [FeFe] hydrogenases and is, therefore, also unlikely to be the site of electron bifurcation (11). Structural comparison of the HydA subunit (of HydABC) with the non-bifurcating $[\mathrm{FeFe}]$ hydrogenase $C p \mathrm{I}$ also reveals that the primary and secondary coordination spheres of the $\mathrm{H}$-cluster are highly conserved in the two enzymes, thereby, supporting our previous conclusion.

Having excluded the H-cluster and FMN, we propose that the bifurcation site is an iron-sulfur cluster or a group of iron-sulfur clusters and are now able to provide two potential mechanisms, both involving the HydB-CT domain. This domain, carrying the B3 and B4 clusters, is found in all characterized electron-bifurcating $[\mathrm{FeFe}]$ hydrogenases but is absent in non-bifurcating NAD ${ }^{+}$ dependent multimeric $[\mathrm{FeFe}]$ hydrogenases $(29,30)$. Therefore, these clusters are considered an essential component of the mechanism. In Mechanism 1 (Fig. 5A), $\mathrm{H}_{2}$ is oxidized by the H-cluster and electrons are sent down the core electron transfer pathway to the FMN. Meanwhile electrons in the adjacent protomer are picked up by cluster A'5, through a large scale conformational change and transferred to the $\mathrm{B} 3 / \mathrm{B} 4$ clusters in the bridge. Another conformational change moves the $\mathrm{B} 3 / \mathrm{B} 4$ clusters close to the $\mathrm{B} 1 / \mathrm{C} 1$ clusters for electron transfer to ferredoxin while $\mathrm{NAD}^{+}$is reduced to $\mathrm{NADH}$ at the FMN. In Mechanism 2 (Fig. 5B), electrons from $\mathrm{H}_{2}$ oxidation at the $\mathrm{H}$ cluster travel via the core electron transfer pathway to $\mathrm{FMN} / \mathrm{C} 1$ and then to the B3/B4 clusters in 
the bridge causing the bridge to close. This then allows a second $\mathrm{H}_{2}$ oxidation to reduce the FMN. $\mathrm{NAD}^{+}$reduction triggers the bridge to reopen moving the reduced $\mathrm{B} 3 / \mathrm{B} 4$ clusters close to the $\mathrm{B} 1 / \mathrm{C} 1$ pair allowing electron transfer to ferredoxin.

\section{A) Mechanism 1}

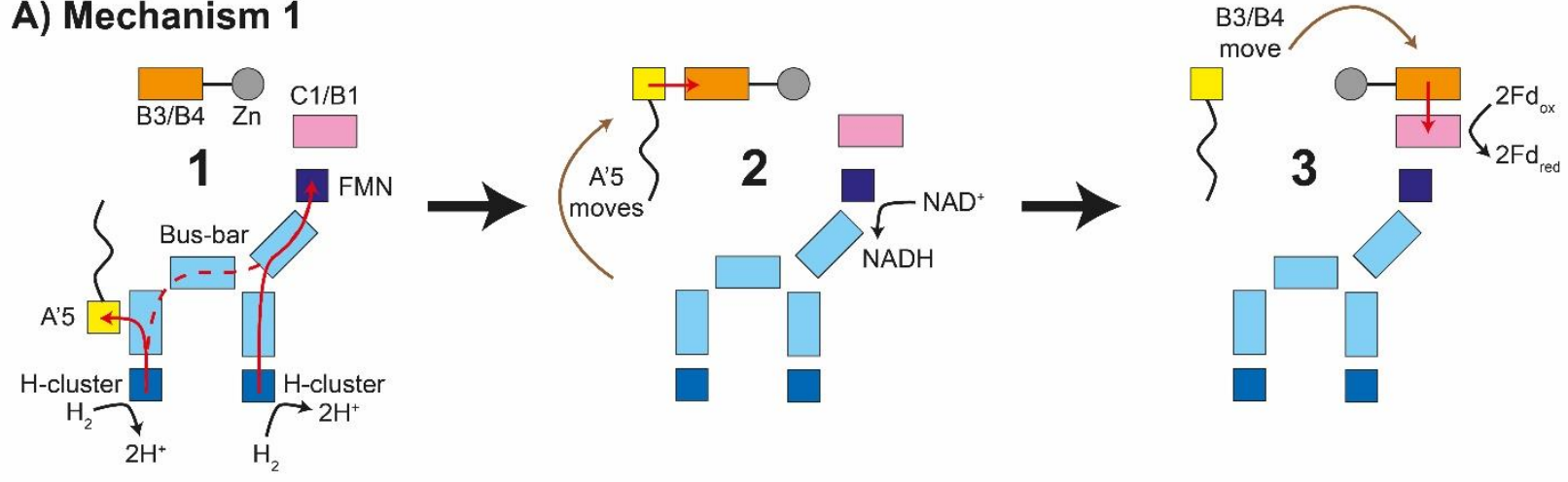

\section{B) Mechanism 2}

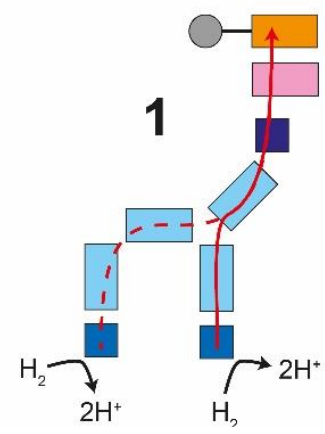

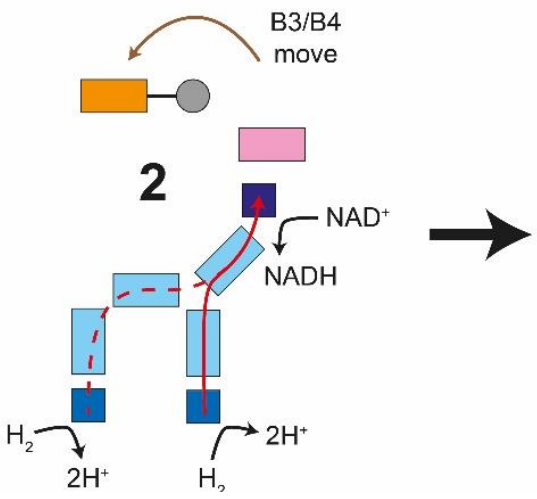

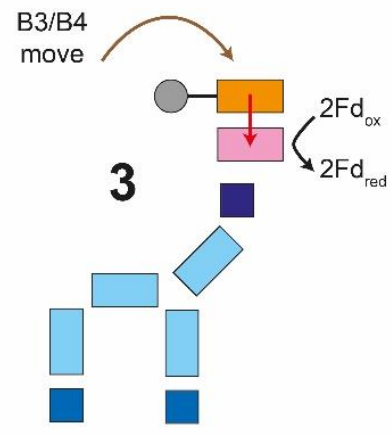

Fig. 5 Illustration of two possible mechanisms of electron transfer in HydABC. A) In Mechanism 1, electrons from $\mathrm{H}_{2}$ oxidation at the H-cluster are transferred to the FMN, via the core electron transfer pathway and cluster A'5 (step 1). A large conformational change moves A' 5 close to the B3/B4 cluster pair in the bridge allowing electron transfer (step 2). This needs to occur twice to put two electrons in the B3/B4 pair. At the same time, $\mathrm{NAD}^{+}$binding and hydride transfer at the FMN forms NADH, triggering a conformational change in the bridge, moving the B3/B4 clusters close enough to the B1/C1 clusters for electron transfer (step 3). Ferredoxin can then accept the electrons from B1. B) In Mechanism 2, electrons generated by oxidation of $\mathrm{H}_{2}$ at the $\mathrm{H}$-cluster travel down the core electron transfer pathway to the FMN and $\mathrm{C} 1$ cluster, and then to the $\mathrm{B} 3 / \mathrm{B} 4$ cluster pair (step 1) closing the bridge (step 2). Further $\mathrm{H}_{2}$ oxidation reduces the FMN leading to $\mathrm{NAD}^{+}$reduction to NADH. This triggers bridge movement allowing the $\mathrm{B} 3 / \mathrm{B} 4$ clusters to transfer electrons to the B1/C1 clusters and then to ferredoxin. Red arrows indicate electron transfer, brown arrows indicate domain movement, and black arrows indicate a catalytic step.

In summary, our cryo-EM structure reveals essential information on the arrangement of cofactors and active sites within T. maritima $\mathrm{HydABC}$, including inter-protomer electronic wiring. Using symmetry expansion, we have also observed two conformations of the HydB-CT domain, a domain that is conserved in bifurcating hydrogenases, consistent with mechanistically relevant conformational changes. By resolving these crucial structural details, the mechanism of bifurcation can now be tackled using site directed mutagenesis coupled to kinetic and spectroscopic studies. These results may also be applied to other enzymes homologous to HydABC. 


\section{References}

1. C. E. Wise, A. E. Ledinina, J. L. Yuly, J. H. Artz, C. E. Lubner, The role of thermodynamic features on the functional activity of electron bifurcating enzymes. Biochim. Biophys. Acta-Bioenerg. 1862, 148377 (2021).

2. P. Rich, The cost of living. Nature. 421, 583 (2003).

3. E. Darrouzet, C. C. Moser, P. L. Dutton, F. Daldal, Large scale domain movement in cytochrome bc1: A new device for electron transfer in proteins. Trends Biochem. Sci. 26, 445-451 (2001).

4. J. W. Peters, D. N. Beratan, G. J. Schut, M. W. W. Adams, On the nature of organic and inorganic centers that bifurcate electrons, coupling exergonic and endergonic oxidation-reduction reactions. Chem. Commun. 54, 4091-4099 (2018).

5. J. W. Peters, A. F. Miller, A. K. Jones, P. W. King, M. W. W. Adams, Electron bifurcation. Curr. Opin. Chem. Biol. 31, 146-152 (2016).

6. V. Müller, N. P. Chowdhury, M. Basen, Electron bifurcation: a long-hidden energy-coupling mechanism. Annu. Rev. Microbiol. 72, 331-353 (2018).

7. A. M. G. Costas, S. Poudel, A. F. Miller, G. J. Schut, R. N. Ledbetter, K. R. Fixen, L. C. Seefeldt, M. W. W. Adams, C. S. Harwood, E. S. Boyd, J. W. Peters, Defining electron bifurcation in the electrontransferring flavoprotein family. J. Bacteriol. 199, e00440-17 (2017).

8. G. J. Schut, M. W. W. Adams, The iron-hydrogenase of Thermotoga maritima utilizes ferredoxin and NADH synergistically: A new perspective on anaerobic hydrogen production. J. Bacteriol. 191, 44514457 (2009).

9. W. Buckel, R. K. Thauer, Flavin-based electron bifurcation, ferredoxin, flavodoxin, and anaerobic respiration with protons $(\mathrm{Ech})$ or $\mathrm{NAD}^{+}(\mathrm{Rnf})$ as electron acceptors: a historical review. Front. Microbiol. 9, 401 (2018).

10. W. Buckel, R. K. Thauer, Energy conservation via electron bifurcating ferredoxin reduction and proton/ $\mathrm{Na}^{+}$translocating ferredoxin oxidation. Biochim. Biophys. Acta - Bioenerg. 1827, 94-113 (2013).

11. N. Chongdar, K. Pawlak, O. Rüdiger, E. J. Reijerse, P. Rodríguez-Maciá, W. Lubitz, J. A. Birrell, H. Ogata, Spectroscopic and biochemical insight into an electron-bifurcating [FeFe] hydrogenase. J. Biol. Inorg. Chem. 25, 135-149 (2020).

12. M. Świerczek, E. Cieluch, M. Sarewicz, A. Borek, C. C. Moser, P. L. Dutton, A. Osyczka, An

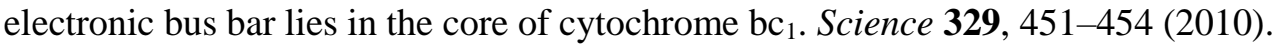

13. R. Baradaran, J. M. Berrisford, G. S. Minhas, L. A. Sazanov, Crystal structure of the entire respiratory complex I. Nature. 494, 443-448 (2013).

14. M. F. J. M. Verhagen, T. O'Rourke, M. W. W. Adams, The hyperthermophilic bacterium, Thermotoga maritima, contains an unusually complex iron-hydrogenase: amino acid sequence analyses versus biochemical characterization. Biochim. Biophys. Acta - Bioenerg. 1412, 212-229 (1999).

15. M. F. J. M. Verhagen, T. W. O'Rourke, A. L. Menon, M. W. W. Adams, Fe-only hydrogenase from Thermotoga maritima. Methods Enzymol. 331, 216-226 (2001).

16. S. M. Ireland, A. C. R. Martin, ZincBind - The database of zinc binding sites. Database. 2019, baz006 (2019).

17. C. C. Page, C. C. Moser, X. Chen, P. L. Dutton, Natural engineering principles of electron tunnelling in biological oxidation-reduction. Nature. 402, 47-52 (1999).

18. J. M. Schuller, J. A. Birrell, H. Tanaka, T. Konuma, H. Wulfhorst, N. Cox, S. K. Schuller, J. Thiemann, W. Lubitz, P. Sétif, T. Ikegami, B. D. Engel, G. Kurisu, M. M. Nowaczyk, Structural adaptations of photosynthetic complex I enable ferredoxin-dependent electron transfer. Science 363, 257-260 (2019).

19. M. Maeda, Y. H. Lee, T. Ikegami, K. Tamura, M. Hoshino, T. Yamazaki, M. Nakayama, T. Hase, Y. Goto, Identification of the $\mathrm{N}$ - and C-terminal substrate binding segments of ferredoxin-NADP ${ }^{+}$ reductase by NMR. Biochemistry. 44, 10644-10653 (2005).

20. N. Cassan, B. Lagoutte, P. Sétif, Ferredoxin-NADP ${ }^{+}$reductase: Kinetics of electron transfer, transient intermediates, and catalytic activities studied by flash-absorption spectroscopy with isolated photosystem I and ferredoxin. J. Biol. Chem. 280, 25960-25972 (2005). 
21. C. Radon, G. Mittelstädt, B. R. Duffus, J. Bürger, T. Hartmann, T. Mielke, C. Teutloff, S. Leimkühler, P. Wendler, Cryo-EM structures reveal intricate Fe-S cluster arrangement and charging in Rhodobacter capsulatus formate dehydrogenase. Nat. Commun. 11, 1-9 (2020).

22. K. Zuchan, F. Baymann, C. Baffert, M. Brugna, W. Nitschke, The dyad of the Y-junction- and a flavin module unites diverse redox enzymes. Biochim. Biophys. Acta-Bioenerg. 1862, 148401 (2021).

23. J. Gutiérrez-Fernández, K. Kaszuba, G. S. Minhas, R. Baradaran, M. Tambalo, D. T. Gallagher, L. A. Sazanov, Key role of quinone in the mechanism of respiratory complex I. Nat. Commun. 11, 4135 (2020).

24. G. J. Schut, E. S. Boyd, J. W. Peters, M. W. W. Adams, The modular respiratory complexes involved in hydrogen and sulfur metabolism by heterotrophic hyperthermophilic archaea and their evolutionary implications. FEMS Microbiol. Rev. 37, 182-203 (2013).

25. J. A. Birrell, K. Morina, H. R. Bridges, T. Friedrich, J. Hirst, Investigating the function of [2Fe-2S] cluster N1a, the off-pathway cluster in complex I, by manipulating its reduction potential. Biochem. J. 456, 139-146 (2013).

26. E. Gnandt, J. Schimpf, C. Harter, J. Hoeser, T. Friedrich, Reduction of the off-pathway iron-sulphur cluster N1a of Escherichia coli respiratory complex I restrains NAD ${ }^{+}$dissociation. Sci. Rep. 7, 8754 (2017).

27. J. H. Artz, D. W. Mulder, M. W. Ratzloff, C. E. Lubner, O. A. Zadvornyy, A. X. Levan, S. G. Williams, M. W. W. Adams, A. K. Jones, P. W. King, J. W. Peters, Reduction potentials of [FeFe]-hydrogenase accessory iron-sulfur clusters provide insights into the energetics of proton reduction catalysis. J. Am. Chem. Soc. 139, 9544-9550 (2017).

28. M. Maldonado, F. Guo, J. A. Letts, A. P. Carter, Atomic structures of respiratory complex III 2 , complex IV, and supercomplex $\mathrm{III}_{2}$-IV from vascular plants. Elife. 10, 1-34 (2021).

29. N. A. Losey, F. Mus, J. W. Peters, H. M. Le, M. J. McInerney, Syntrophomonas wolfei uses an NADHdependent, ferredoxinindependent [FeFe]-hydrogenase to reoxidize NADH. Appl. Environ. Microbiol. 83, e01335-17 (2017).

30. N. A. Losey, S. Poudel, E. S. Boyd, M. J. McInerney, The beta subunit of non-bifurcating NADHdependent [FeFe]-hydrogenases differs from those of multimeric electron-bifurcating [FeFe]hydrogenases. Front. Microbiol. 11, 1109 (2020).

31. W. Buckel, R. K. Thauer, Flavin-based electron bifurcation, a new mechanism of biological energy coupling. Chem. Rev. 118, 3862-3886 (2018).

32. J. Zivanov, T. Nakane, B. O. Forsberg, D. Kimanius, W. J. H. Hagen, E. Lindahl, S. H. W. Scheres, New tools for automated high-resolution cryo-EM structure determination in RELION-3. Elife. 7, e42166 (2018).

33. A. Rohou, N. Grigorieff, CTFFIND4: Fast and accurate defocus estimation from electron micrographs. J. Struct. Biol. 192, 216-221 (2015).

34. J. Zivanov, T. Nakane, S. H. W. Scheres, Estimation of high-order aberrations and anisotropic magnification from cryo-EM data sets in RELION-3.1. IUCrJ. 7, 253-267 (2020).

35. N. W. Moriarty, P. D. Adams, Iron-sulfur clusters have no right angles. Acta Crystallogr. Sect. D Struct. Biol. 75, 16-20 (2019).

36. M. Nouailler, X. Morelli, O. Bornet, B. Chetrit, Z. Dermoun, F. Guerlesquin, Solution structure of HndAc: A thioredoxin-like domain involved in the NADP-reducing hydrogenase complex. Protein Sci. 15, 1369-1378 (2006).

37. L. A. Kelley, S. Mezulis, C. M. Yates, M. N. Wass, M. J. E. Sternberg, The Phyre2 web portal for protein modeling, prediction and analysis. Nat. Protoc. 10, 845-858 (2015).

38. J. M. Kuchenreuther, C. S. Grady-Smith, A. S. Bingham, S. J. George, S. P. Cramer, J. R. Swartz, High-yield expression of heterologous [FeFe] hydrogenases in Escherichia coli. PLoS One. 5, e15491 (2010).

39. G. Berggren, A. Adamska, C. Lambertz, T. R. Simmons, J. Esselborn, M. Atta, S. Gambarelli, J. M. Mouesca, E. Reijerse, W. Lubitz, T. Happe, V. Artero, M. Fontecave, Biomimetic assembly and activation of [FeFe]-hydrogenases. Nature. 499, 66-69 (2013).

40. J. Esselborn, C. Lambertz, A. Adamska-Venkatesh, T. Simmons, G. Berggren, J. Noth, J. Siebel, A. 
Hemschemeier, V. Artero, E. Reijerse, M. Fontecave, W. Lubitz, T. Happe, Spontaneous activation of [FeFe]-hydrogenases by an inorganic [2Fe] active site mimic. Nat. Chem. Biol. 9, 607-609 (2013).

41. K. D. Swanson, M. W. Ratzloff, D. W. Mulder, J. H. Artz, S. Ghose, A. Hoffman, S. White, O. A. Zadvornyy, J. B. Broderick, B. Bothner, P. W. King, J. W. Peters, [FeFe]-hydrogenase oxygen inactivation is initiated at the H cluster 2Fe subcluster. J. Am. Chem. Soc. 137, 1809-1816 (2015).

42. J. Esselborn, N. Muraki, K. Klein, V. Engelbrecht, N. Metzler-Nolte, U. P. Apfel, E. Hofmann, G. Kurisu, T. Happe, A structural view of synthetic cofactor integration into [FeFe]-hydrogenases. Chem. Sci. 7, 959-968 (2016).

43. A. J. Noble, V. P. Dandey, H. Wei, J. Brasch, J. Chase, P. Acharya, Y. Z. Tan, Z. Zhang, L. Y. Kim, G. Scapin, M. Rapp, E. T. Eng, W. J. Rice, A. Cheng, C. J. Negro, L. Shapiro, P. D. Kwong, D. Jeruzalmi, A. des Georges, C. S. Potter, B. Carragher, Routine single particle CryoEM sample and grid characterization by tomography. Elife. 7, e34257 (2018).

44. J. Zivanov, T. Nakane, S. H. W. Scheres, A Bayesian approach to beam-induced motion correction in cryo-EM single-particle analysis. IUCrJ. 6, 5-17 (2019).

45. S. L. Ilca, A. Kotecha, X. Sun, M. M. Poranen, D. I. Stuart, J. T. Huiskonen, Localized reconstruction of subunits from electron cryomicroscopy images of macromolecular complexes. Nat. Commun. 6 , 8843 (2015).

\section{Acknowledgments}

This work benefited from access to the Astbury Biostructure Laboratory, an Instruct-ERIC centre. Financial support was provided by Instruct-ERIC (PID 11666). We are grateful to Svetomir Tzokov, Charlotte Scarff, and Rebecca Thompson for assistance with data collection and Nigel Moriarty for assistance with FeS ligation during model building. We used computational resources provided by the Viking Cluster at the University of York and are grateful to the Research Computing team and Huw Jenkins for assistance with computing. We thank Mikroanalytisches Laboratorium Kolbe for ICP-MS measurements. We are grateful to Hannah Bridges, Laure Decamps and Patrícia Rodríguez Maciá for critical evaluation of the manuscript. J.A.B. and N.C. acknowledge funding from the DFG SPP 1927 "Iron-Sulfur for Life" project (Project No. BI 2198/1-1). The work was supported by the Max Planck Society (J.A.B., N.C. and W.L.), and in part a UKRI Future Leader Fellowship (JNB; MR/T040742/1) and JSPS KAKENHI (Grant number 16K21748 (H.O.).

\section{Author contributions}

Conceptualization: NC, HO, WL, JNB, JAB

Methodology: CF, NC, PG, JNB, JAB

Investigation: $\mathrm{CF}, \mathrm{NC}, \mathrm{PG}, \mathrm{JNB}, \mathrm{JAB}$

Visualization: CF, PG, JNB

Funding acquisition: JNB, JAB

Project administration: JAB, JNB

Supervision: JAB, JNB

Writing - original draft: $\mathrm{CF}, \mathrm{NC}, \mathrm{JNB}, \mathrm{JAB}$

Writing - review \& editing: $\mathrm{CF}, \mathrm{NC}, \mathrm{PG}, \mathrm{HO}, \mathrm{WL}, \mathrm{JNB}, \mathrm{JAB}$

\section{Competing interests}

Authors declare that they have no competing interests.

\section{Data and materials availability}

Protein databank (PDB) files for the four model presented in this manuscript are available at https://www.rcsb.org/ under PDB ID 7P5H (D2 tetramer, 7P8N (Bridge closed forward), 7P91 
(Bridge closed reverse), and 7P92 (Open bridge). Cryo-EM maps are available at https://www.ebi.ac.uk/pdbe/emdb/. All other data are available in the main text or the supplementary materials.

\section{Supplementary Materials}

Materials and Methods

Supplementary Text

Figs. S1 to S8

Tables S1 